\title{
Muscle synergies in preparation to a step made with obstacle in elderly individuals
}

\author{
Yun Wang ${ }^{1 *}$, Kazuhiko Watanabe ${ }^{2}$ and Tadayoshi Asaka ${ }^{3}$
}

\begin{abstract}
Background: To evaluate if multi-muscle synergies are comprised of flexible combinations of a small number of postural muscles to stabilize the center of pressure (COP) shift during preparation to making a step in the elderly (self-paced level stepping vs. obstacle crossing stepping).

Methods: Electromyography (EMG) signals of leg and trunk muscles were recorded. Linear combination of integrated indices of muscle activity (M-modes) and their relationship to changes in the COP shift in the anterior-posterior (AP) direction were first determined. Uncontrolled manifold (UCM) analysis was performed to determine the extent to which variance of the M-modes acted to produce a consistent change in the COP displacement.

Results: The elderly were capable of stabilizing the COPAP coordinate based on co-varied involvement of the M-modes. The synergy index $(\Delta \mathrm{V})$ changes in the elderly emerged later (100 ms prior to $\left.t_{0}\right)$ and its magnitude was smaller as compared to that reported in younger persons.

Conclusions: Our study reveals that aging is associated with a preserved ability to explore the flexibility of the M-mode compositions but a decrease ability to use multi-M-mode synergies following a predictable perturbation.
\end{abstract}

Keywords: Anticipatory synergy adjustments, Synergies, Step initiation, Obstacle, Elderly

\section{Background}

Stepping over an obstacle is a complex task that requires translating the center of mass closer to the edge of the base of support, with large inertial forces that could potentially threaten stability. Because the sensory and motor resources that are required for postural stability decline with age, stepping over an obstacle can become quite a demanding task and pose a great risk in older adults [1,2]. Moreover, aging is associated with major changes in the neuromotor system. In particular, these include reduction in muscle strength, power and joint mobility along with an impaired sensorimotor integration. All these changes can potentially contribute to deterioration of postural control and mobility in the elderly. Previous studies have shown that lower accuracy and higher variability in obstacle crossing tasks by elderly subjects $[3,4]$.

\footnotetext{
* Correspondence: yunwang70@hotmail.com

${ }^{1}$ Tianjin Key Lab of Exercise Physiology and Sports Medicine, Department of Health and Exercise Science, Tianjin University of Sport, 51 Weijin South Street, Hexi District, Tianjin 300381, China

Full list of author information is available at the end of the article
}

Wang et al. investigated the multi-muscle synergies used by healthy young participants to stabilize the anterior-posterior (AP) trajectory of the center of pressure (COP) during preparation to making a step with obstacle [5]. Within the obstacle-negotiation paradigm, stepping over obstacle task from quiet stance was combined to comfortable level stepping. These different tasks were designed to study the different organizations of leg and trunk muscles into groups (M-modes) and trial-to-trial co-variations of M-mode involvement (M-mode synergies) during stepping tasks, using the uncontrolled manifold (UCM) analysis. The UCM analysis assumes that the neural controller acts in a space of independent elemental variables (for example, electromyographic signals, EMGs) and creates in that space a sub-space (UCM) corresponding to a value or a time profile of a specific performance variable (for example, COP trajectory), which is assumed to be important for postural tasks. It begins with identification of M-modes with parallel scaling of muscle activation levels [6-10]. Then, the COP shifts are mapped on small changes in the M-modes magnitudes, resulting in a Jacobian matrix. Further, co-variation of M-modes magnitudes is analyzed 
to quantify synergies stabilizing the COP coordinate [9]. Wang et al. found that most of the across-trials variance in the $\mathrm{M}$-modes space stabilized the average value of $\mathrm{COP}_{\mathrm{AP}}$ shift ("good variance"; within UCM, $\mathrm{V}_{\mathrm{UCM}}$ ) whereas the component of the M-modes space variance ("bad variance"; orthogonal to $\mathrm{UCM}, \mathrm{V}_{\mathrm{ORT}}$ ) resulting in $\mathrm{COP}_{\mathrm{AP}}$ variability was smaller. As such, these findings highlight the importance of M-modes in control of posture, and point out the existence of the robustness of multi-M-mode synergies across different manners of making a step.

While such coordination pattern in the activation of the lower extremities and the trunk muscles are established in the healthy young adults [5-10], to the best of our knowledge there are no studies that evaluate the effect of aging on the multi-M-mode synergies during the stepping over obstacle task. Given that aging is associated with a decline in muscle mass, strength, coordination and postural stability $[11,12]$, comprehending the age-related differences in the muscle synergies during preparation to making a step with obstacle is vital. While the impairments of anticipatory postural control in the elderly have been demonstrated $[13,14]$, it is not specifically known how these changes influence multi muscle coordination in controlling the body's COP displacement closer to the boundaries of the base of support, that compromising balance. The outcome of a recent study on postural control in step initiation revealed that there were M-mode synergies stabilizing COP shifts in the stepping and supporting legs in the young and elderly subjects. However, the synergies of the older adults showed a reduced and delayed value than that of the young persons [15]. These findings indicate that when initiating gait from a quiet standing position, older adults may have difficult in utilizing anticipatory postural synergies. As such, it is important to investigate whether older adults are able to effectively utilize preparatory muscle coordination in balance maintenance prior to making a step with obstacle.

Therefore, the objective of the present study was focused on examining possible changes of multi-M-mode synergies during preparation to making a step in elderly individuals. We hypothesized that multi-M-mode synergies would be observed during the task of stepping over obstacle. In particular, we expected to see that the synergies of the older adults will be delayed and reduced in magnitude and will be associated with greater COP displacements in the anterior-posterior (AP) direction.

\section{Methods}

\section{Subjects}

Nine healthy older adults (five males and four females; mean age $=72.3 \pm 4.4 \mathrm{yr}$, mean weight $=59.0 \pm 9.2 \mathrm{~kg}$, and mean height $=160.0 \pm 5.2 \mathrm{~cm}$ ) participated in the study. None of the subjects had a balance disorder or experienced dizziness, and none of them had neurological or musculoskeletal disorders. All of the subjects were right-foot-dominant according to preferred foot usage when kicking a ball, stepping up on a chair, and leaping off in the long jump [16]. All of the subjects gave their informed consent to take part in the study, which is consistent with the 1964 Declaration of Helsinki.

\section{Experimental protocol}

To test the effect of aging on the ability of older adults to change the multi-muscle synergies in preparation to a step made with obstacle, we presented subjects with an obstacle set at $15 \%$ of the subject's body height (Figure 1, A). A lightweight PVC pipe crossbar was used as the obstacle. The choice of the obstacle height was based on the following considerations. This task was used in our earlier studies of obstacle crossing $[5,17,18]$ and the same high obstacle height on older subjects has been used in previous researches [2,3] to reflect typical high height encountered in everyday life. The obstacle location was chosen in any distance by each subject.

The experiment included two parts (control-test and stepping-test). The control-test involved two tasks (Figure 1, $B$ and C): (1) quiet standing while holding steadily a load of $5.3 \mathrm{~kg}$ in front of the body through the pulley system ( $Q S_{L F}$ task); (2) quiet standing while holding steadily a load of $5.3 \mathrm{~kg}$ behind the body $\left(Q S_{L B}\right.$ task) through the pulley system. When the subjects were facing the pulley, they counteracted the load by activating the dorsal muscles of the leg and trunk muscles; when they were facing away from the pulley, the ventral muscles were activated. For each task, the subjects were required to stand as still as possible and keep the body vertical for $5 \mathrm{~s}$. The control-test was used to normalize the EMG signals for individual subjects. The stepping-test involved two tasks: (1) comfortable stepping task $\left(S T_{C S}\right),(2)$ obstacle stepping task $\left(S T_{O S}\right)$. Before the stepping-test, 2-5 practice trials were given to all subjects for familiarization with the task.

The stepping-test required the subjects to step forward with the right leg and followed with the left leg so that both feet came to rest forward. In the $S T_{C S}$ task, the subjects were asked to make a comfortable level step from quiet stance in a self-paced manner. In the $S T_{O S}$ task, the subjects were required to step over an obstacle of $15 \%$ body height from quiet stance in a self-paced way [5]. For each task, the subjects were instructed to look straight ahead and with no restrictions to the arms. The tasks were performed in two blocks of 20 trials. There were at least a 6-s interval between trials and a 2-min interval between tasks, to avoid fatigue. Foot position was marked on the top of the platform to keep the same position across all the trials. The tasks were performed in a random order across subjects. 

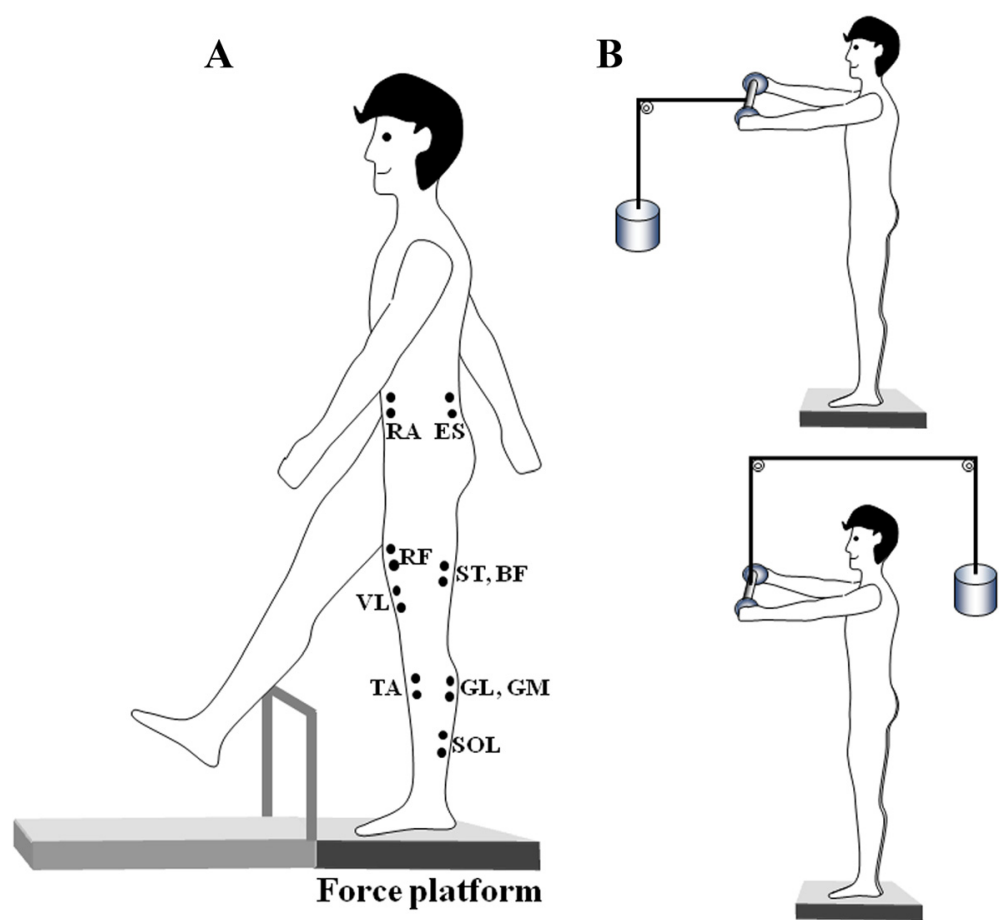

Figure 1 The experimental setup. A. The subjects were required to step over an obstacle of 15\% body height from quiet stance in a self-paced way. B. In the control trials, the subjects were required to hold a load $(5.3 \mathrm{Kg})$ in front of the body or behind the body for $5 \mathrm{~s}$, they held a handle which was connected to the load through a pulley system. Location of some of the EMG electrodes is also shown in panel A (GL lateral head of gastrocnemius, GM medial head of gastrocnemius, SOL soleus, ST semi-tendinosus, BF biceps femoris, GMED gluteus medius, ES erector spinae, $T A$ tibialis anterior, $V L$ vastus lateralis, $R F$ rectus femoris, $T F$ tensor fasciae latae, $R A$ rectus abdominis).

\section{Data collection}

In all tasks, the subjects stood barefoot on a force platform (AMTI, Watertown, MA; Model BP400600-2000) with their feet shoulder-width apart, eyes open. The vertical component of the ground reaction force $\left(\mathrm{F}_{\mathrm{Z}}\right)$, the horizontal component of the ground reaction force in the anteriorposterior direction $\left(\mathrm{F}_{\mathrm{X}}\right)$, and the moment of force about the frontal-horizontal axis $\left(M_{Y}\right)$ were recorded. Electromyography (EMG) activity was recorded from ten lower limb and trunk muscles of the subject's right side. After the skin was shaved and cleaned with alcohol, active surface EMG electrodes (Biometrics, United Kingdom) were placed on the muscle bellies. The following muscles were recorded: tibialis anterior (TA), lateral head of gastrocnemius (GL), medial head of gastrocnemius (GM), soleus (SOL), rectus femoris (RF), vastus lateralis (VL), biceps femoris (BF), semitendinosus (ST), rectus abdominis (RA) and erector spinae (ES). Experimental data were digitized at the sampling frequency of $1000 \mathrm{~Hz}$ with a 16-bit resolution. A foot switch was placed under the heads of the metatarsal bones of right foot to measure the timing of toe off.

\section{Data processing}

The data were processed offline using MATLAB Version 8.0 (R2012b, The MathWorks, Natick, MA) software packages. Raw EMG data were rectified and filtered using fourth-order Butterworth low-pass filter with a $50-\mathrm{Hz}$ cutoff frequency. Signals from the force plate were low-pass filtered at $20 \mathrm{~Hz}$. Coordinates of the COP in the anterior-posterior (AP) direction were calculated based on the formula:

$$
\mathrm{COP}_{\mathrm{AP}}=\left(-\mathrm{M}_{\mathrm{Y}}+\left(\mathrm{F}_{\mathrm{X}} \times \mathrm{d}\right)\right) / \mathrm{F}_{\mathrm{Z}}
$$

where coefficient $d$ is the distance from the origin of the force platform to its top surface $(0.045 \mathrm{~m}$ according to the manufacturer's specifications) [19].

The 'time zero' $\left(t_{0}\right)$ was defined by the toe off time using the signal from the foot switch. Rectified EMG signals were integrated over $25 \mathrm{~ms}$ intervals in a time window from $-500 \mathrm{~ms}$ (before $t_{0}$ ) to $t_{0}$. These EMG integrals for each of $25 \mathrm{~ms}$ were then corrected by subtracting the EMG integrals of the baseline activity during quiet neutral stance in the control trial. The outcome of the adjusted EMG integral will be denoted as IEMG. $\triangle I E M G$ indices were further normalized $\left(\Delta / \mathrm{EMG}_{\mathrm{N}}\right)$ by the EMG integrals collected in the control trials as follows: $\triangle I E M G$ indices for the dorsal (SOL, GL, GM, BF, $\mathrm{ST}$, and ES) muscles were divided by the EMG integrals over $25 \mathrm{~ms}\left(\mathrm{IEMG}_{\mathrm{C}}\right)$ in the middle of the control trial with 
holding the load quietly in front of the body, while $\triangle I E M G$ indices for the ventral (TA, RF, VL, and RA) muscles were divided by the EMG integrals over $25 \mathrm{~ms}\left(I E M G_{C}\right)$ in the middle of the corresponding control trial, that is during holding the load quietly behind the body. Five $100 \mathrm{~ms}$ time windows in relation to $\mathrm{t}_{0}$ were analyzed, from -500 to $-400 \mathrm{~ms}$ (T1), from -400 to $-300 \mathrm{~ms}$ (T2), from -300 to $-200 \mathrm{~ms}$ (T3), from -200 to $-100 \mathrm{~ms}$ (T4) and from $-100 \mathrm{~ms}$ to $\mathrm{t}_{0}$ (T5). Different time intervals were defined based on our prior studies to reflect possible time development of the multi-muscle synergies $[5,7]$.

\section{Defining M-modes and Jacobians}

We extracted groups of muscles (M-modes) from the $I \mathrm{EMG}_{\mathrm{N}}$ data matrix within the time window in relation to $t_{0}$ from $-200 \mathrm{~ms}$ to $t_{0}$ using PCA. For each subject, the $I E M G_{N}$ data formed a matrix of 8 time intervals $\times 10$ muscles $\times 20$ trials $=1600$ data points. The correlation matrix among the IEMG was subjected to principal component analysis with Varimax rotation, using procedures from SPSS (SPSS, Inc., Chicago, Illinois, USA). The factor analysis module with principal component extraction was employed.

For each subject, the obtained eigen-values and PCs were then considered. The first four PCs (described in more detail in Results) were selected for further analysis. This was determined by examining of the scree plots and having at least two muscles significantly loaded per PC. We are going to address these PCs as muscle modes (M-modes) and assume that magnitudes of (coefficients at) the M-modes are manipulated by the controller to produce $\mathrm{COP}_{\mathrm{AP}}$ shifts. A reciprocal M-mode is defined as a pattern with significant loading coefficients on the ventral muscles ("push-back" mode), or on the dorsal muscles ("push-forward" mode), while a co-contraction M-mode is defined as a pattern with significant loading coefficients on the same M-mode with the same sign for two muscles with opposing action at a particular joint [8].

Small changes in the magnitudes of $\mathrm{M}$-modes $(\Delta \mathrm{M})$ were related to the change in the $\mathrm{COP}_{\mathrm{AP}}$ shifts $\left(\triangle \mathrm{COP}_{\mathrm{AP}}\right)$ through the Jacobian $(\mathbf{J})$. Multiple linear regression analysis over the trials was used to define the $\mathbf{J}$ for each subject separately. The $\mathbf{J}$ was estimated as coefficients of multiple linear regression between across-trials $\Delta \mathrm{Ms}$ and $\Delta \mathrm{COP}_{\mathrm{AP}}$.

\section{Computing synergy index: UCM analysis}

For each trial of the $S T_{C S}$ and $S T_{O S}$ tasks, $\triangle \mathrm{IEMG}_{\mathrm{N}}$ were computed and transformed into $\Delta \mathrm{Ms}$ by multiplying the loadings of the individual $\mathrm{M}$-mode. The mean magnitudes of each $\Delta \mathrm{M}$ for a selected time interval across a series of ST trials were computed. Since the model relating $\Delta \mathrm{Ms}$ to $\Delta \mathrm{COP}_{\mathrm{AP}}$ is linear, the mean values were subtracted from each computed value and the residuals were further analyzed.
The UCM represents different combinations of M-modes that keep the value of $\triangle \mathrm{COP}_{\mathrm{AP}}$ unchanged. The UCM was estimated as the null space of the corresponding $\mathbf{J}$ matrix. The null space is spanned by the basis vectors, $\boldsymbol{\varepsilon}_{i}$. The vector of individual mean-free $\Delta \mathrm{Ms}$ was resolved into its projection onto the null space and the orthogonal subspace:

$$
\begin{aligned}
& f_{U C M}=\sum_{i=1}^{n-d}\left(\varepsilon_{i}^{T} \cdot(\Delta M)\right) \varepsilon_{i} \\
& f_{\text {ORT }}=(\Delta M)-f_{U C M}
\end{aligned}
$$

where $\mathrm{n}=4$ and $\mathrm{d}=1$ are the number of dimensions of the UCM and of task space.

Variance per degree of freedom within the UCM and orthogonal to the UCM across trials were computed as:

$$
\begin{aligned}
& V_{U C M}=\sigma_{U C M}^{2}=\sum_{i=1}^{N} f_{U C M}^{2} /\left((n-d) N_{\text {trials }}\right) \\
& V_{O R T}=\sigma_{O R T}^{2}=\sum_{i=1}^{N} f_{O R T}^{2} /\left(d N_{\text {trials }}\right)
\end{aligned}
$$

We computed an index of synergy $(\Delta V)$ reflecting the difference between the variance within the UCM and orthogonal to the UCM:

$$
\Delta V=\left(V_{U C M}-V_{O R T}\right) / V_{T O T}
$$

where all variance indices are computed per degree of freedom; $V_{\text {TOT }}$ means the total variance. For further analyses, the $\Delta V$ values were transformed using a Fisher's z-transformation $\left(\Delta V_{Z}\right)$ adapted to the boundaries of $\Delta V$ :

$$
\Delta V_{Z}=\frac{1}{2} \cdot \log \left[\frac{4+\Delta V}{1 \frac{1}{3}-\Delta V}\right]
$$

\section{Statistics}

The data are presented in the text and figures as mean \pm SD. The fractions of variance explained by the first four principal components were transformed into z-scores using standard Fisher's z-transformation. Paired $t$ test was used for comparing the $\mathrm{z}$-scores and the peak $\mathrm{COP}_{\mathrm{AP}}$ shifts between the two tasks. For the data of $\Delta \mathrm{V}_{\mathrm{Z}}$, two-way ANOVA was used with factors Task $\left(\mathrm{ST}_{\mathrm{CS}}\right.$ and $\mathrm{ST}_{\mathrm{OS}}$ ), Time interval (five intervals) to analyze possible changes in values of $\Delta \mathrm{V}_{\mathrm{Z}}$ across tasks and with time. Tukey post-hoc tests were used where appropriate. For all statistical analyses, p-value less than 0.05 was set as a measure of significance.

\section{Results}

\section{General EMG patterns and COP displacements}

Postural muscles demonstrated similar early changes across the EMG patterns associated with making a step. Figure 2 shows the rectified EMGs of the leg and trunk muscles averaged across trials by a representative subject 


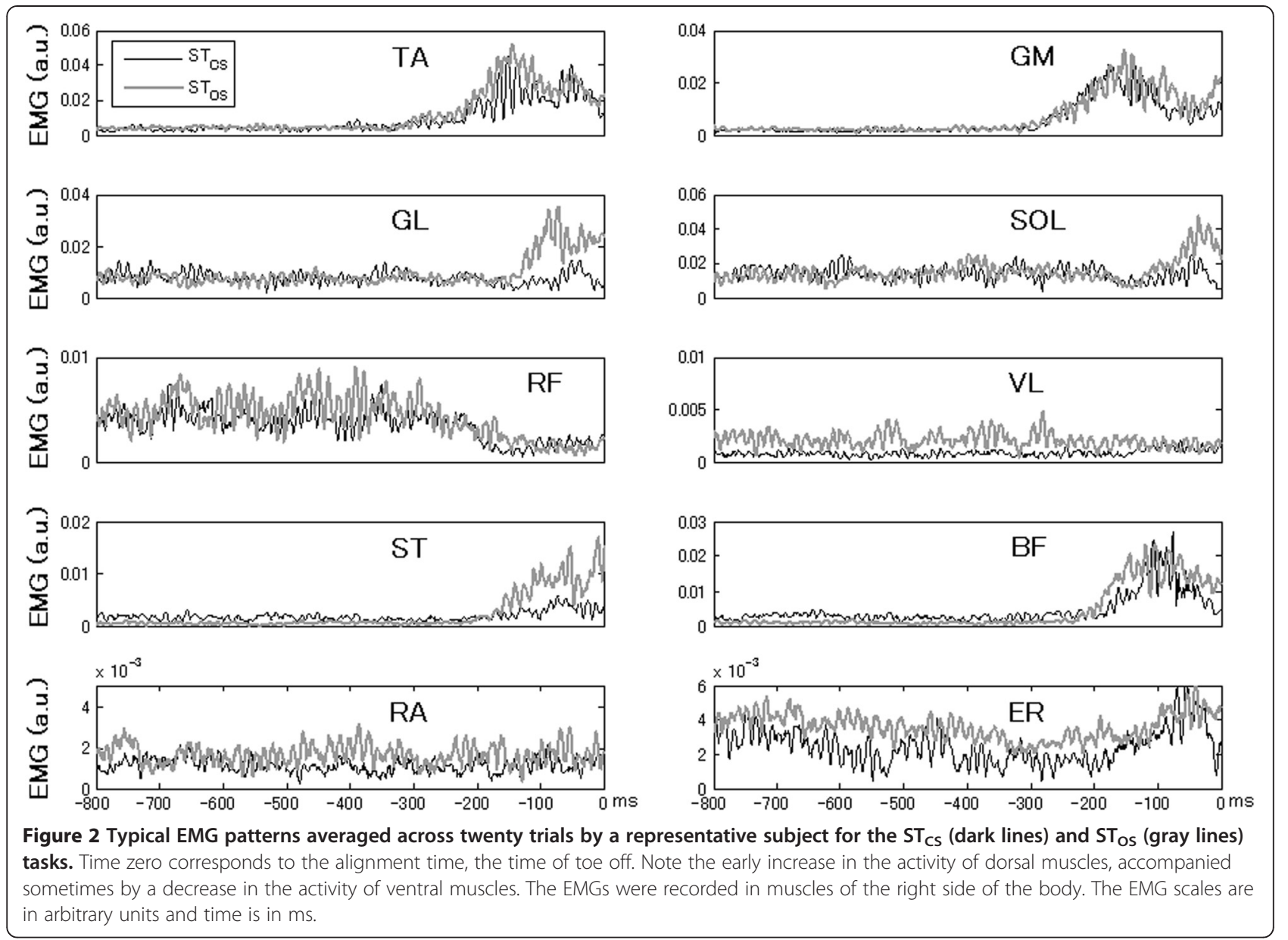

in the $\mathrm{ST}_{\mathrm{CS}}$ and $\mathrm{ST}_{\mathrm{OS}}$ tasks. In the $\mathrm{ST}_{\mathrm{CS}}$ task, the stepping leg typically showed alternating bursts of activity in the ventral and dorsal muscles. The ventral muscles showed a decrease in the baseline activity but the dorsal muscles showed an increase in the activity just before the step initiation. In the $\mathrm{ST}_{\mathrm{OS}}$ task, there was a substantial increase in the level of activity of most muscles. The regularities in the patterns of activation of the leg and trunk muscles were consistently observed in the $\mathrm{ST}_{\mathrm{CS}}$ and $\mathrm{ST}_{\mathrm{OS}}$ tasks. Note that muscle activity in the $\mathrm{ST}_{\mathrm{CS}}$ task was typically higher than that in the $\mathrm{ST}_{\mathrm{OS}}$ task. Muscle activity varied across subjects, and some subjects did not show clear bursts or episodes of EMG suppression in some muscles.

In preparation to stepping, subjects shifted the COP in the AP direction backwards (Figure 3). This adjustment allowed to unload the stepping leg and to create a moment of the reactive force rotating the body forward about the ankle joints. There was larger anticipatory $\mathrm{COP}_{\mathrm{AP}}$ displacement in the $\mathrm{ST}_{\mathrm{CS}}$ task $\left(\mathrm{COP}_{\mathrm{AP}-\mathrm{CS}}=-2.42 \pm 1.22 \mathrm{~cm}\right)$ as compared to the $\mathrm{ST}_{\mathrm{OS}}$ task $\left(\mathrm{COP}_{\mathrm{AP}-\mathrm{OS}}=-1.75 \pm 1.02 \mathrm{~cm}\right)$; the difference was statistically significant $(\mathrm{p}<0.05)$. Negative values correspond to backward displacements.

\section{Principal component analysis and multiple regression analysis}

Indices of integrated muscle activity were measured within the time window in relation to $t_{0}$ from $-200 \mathrm{~ms}$ to $t_{0}$ (see the Methods). The normalized integrated EMG indices $\left(I E M G_{N}\right)$ were subjected to principle component

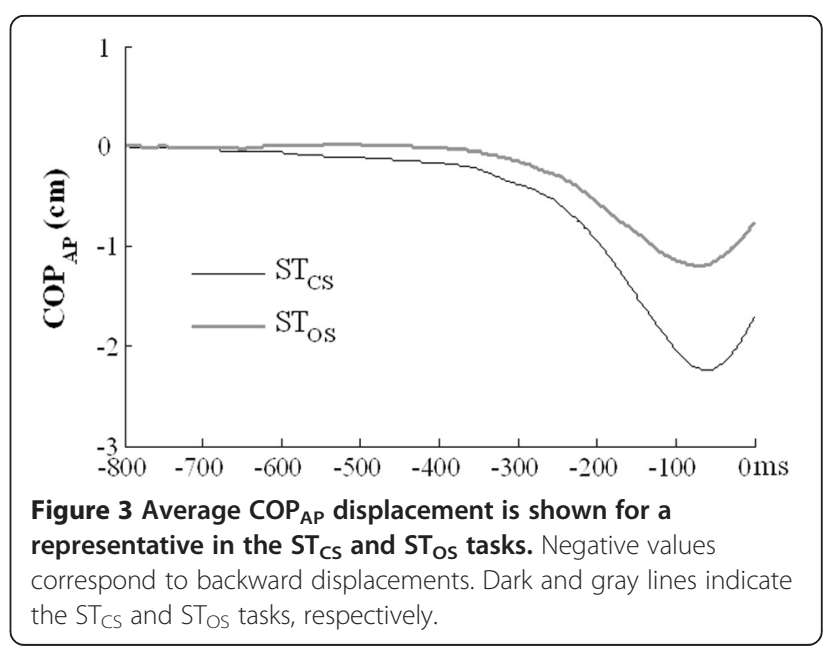


analysis (PCA) with Varimax rotation to identify muscle groups, eigenvectors in the muscle activation space. As a result, the ten-dimensional muscle activation space was reduced to a four-dimensional $\mathrm{M}$-mode space.

On an average, the first four principal components (PCs) accounted for the $62.2 \pm 5.9 \%$ total variance in the muscle activation space in the $\mathrm{ST}_{\mathrm{CS}}$ task and $60.0 \pm 4.2 \%$ in the $\mathrm{ST}_{\mathrm{OS}}$ task. There was considerable variability across the subjects in the M-mode composition. The loadings for all the muscles on the four factors for a representative subject in the $\mathrm{ST}_{\mathrm{CS}}$ task are presented in Figure 4 (top panel). The first M-mode showed high loading values with the same sign for the IEMG $_{N}$ indices of the GL, GM, SOL muscles. The second M-mode showed high loading values for the TA, RF, $\mathrm{VL}$ muscles. The muscles which showed high loading in the third M-mode were the ST and BF muscles. In the fourth M-mode however, the loading pattern were higher for the RA and ES muscles.

It is important to note that the first M-mode revealed a reciprocal contraction of the leg muscles ("push-back" M-mode) and the second M-mode depicted a reciprocal contraction of the thigh and leg muscles ("push-forward"
M-mode). Similarly, significant loading coefficients for the third M-mode revealed a reciprocal contraction of the thigh muscles ("push-back" M-mode). A reciprocal M-mode is defined as a pattern with significant loading coefficients on the ventral muscles ("push-back" mode), or on the dorsal muscles ("push-forward" mode). Significant loading coefficients for the fourth M-mode seen in the RA and ES muscles with opposing actions revealed a co-contraction at the hip joint.

Representative results of the PCA in the $\mathrm{ST}_{\mathrm{OS}}$ task are presented in Figure 4 (bottom panel). The first M-mode composition was a "push-back" M-mode. The second M-mode showed high loading values for two ventral (TA and RF) and one dorsal (BF) muscles acting at the knee joint, while the third M-mode showed high loading values for the RA (ventral) and ES (dorsal) muscles, which is a "co-contraction at the hip" pattern. The fourth M-mode again showed a push-back pattern between the VL and ST muscles, with the opposite sign. Overall, in the $\mathrm{ST}_{\mathrm{CS}}$ task, $10 \mathrm{M}$-modes with "co-contraction" $\mathrm{M}$-mode were seen, while in the $\mathrm{ST}_{\mathrm{OS}}$ task, the number of M-modes with co-contraction patterns was 15 from a total of $36 \mathrm{M}$-modes.

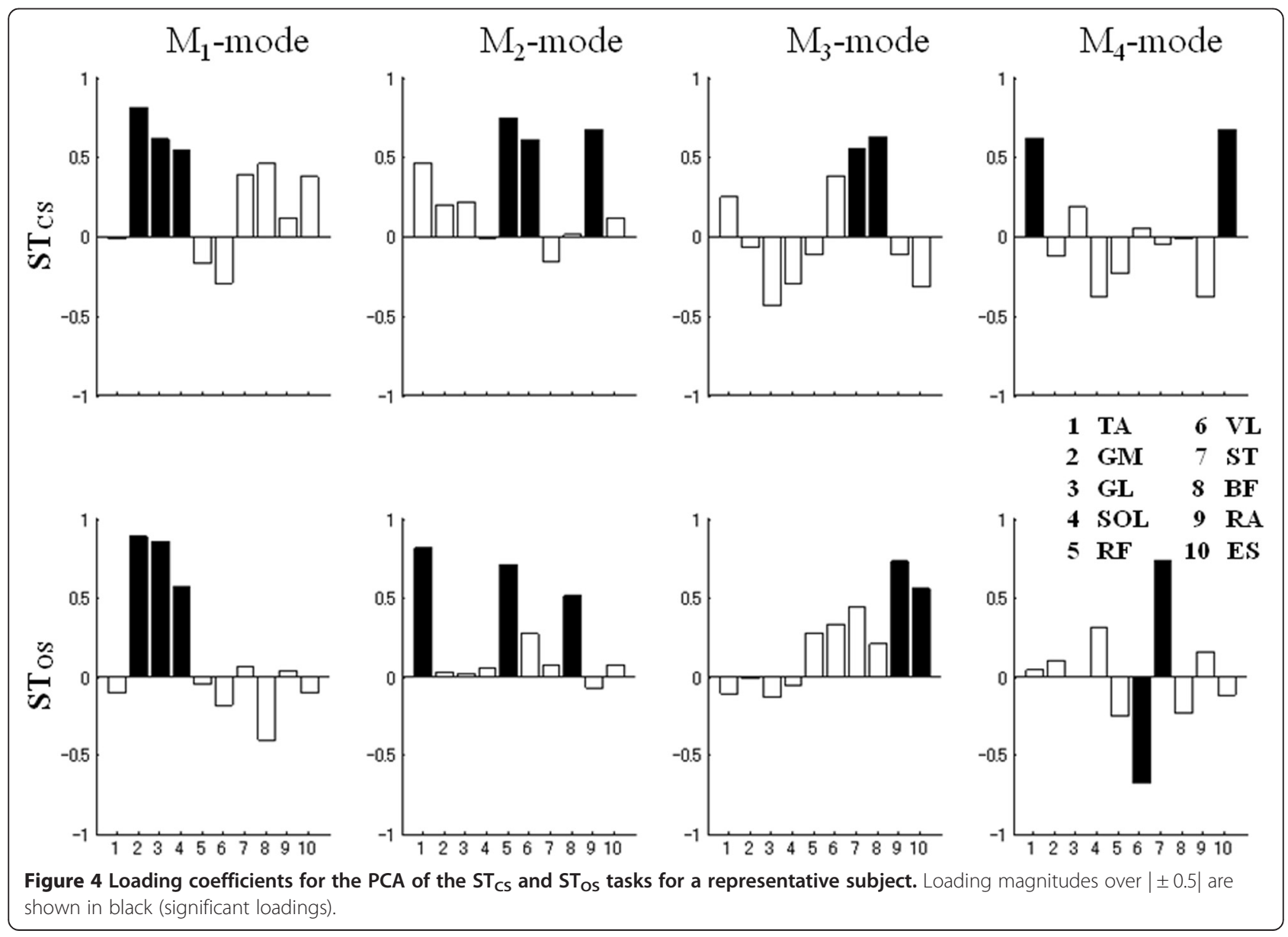


Multiple regression analysis was performed to define the Jacobian mapping small changes in the M-mode magnitudes onto $\mathrm{COP}_{\mathrm{AP}}$ shifts. Results of multiple regression analysis were significant in most cases for each of the two tasks. On an average, the analysis accounted for $85.7 \pm 10.2 \%$ and $85.0 \pm 14.7 \%$ of variance in $\triangle \mathrm{COP}_{\mathrm{AP}}$ in the $\mathrm{ST}_{\mathrm{CS}}$ and $\mathrm{ST}_{\mathrm{OS}}$ tasks respectively. There was no task difference in variations in the magnitudes of the four $\mathrm{M}$-modes accounted for the total variance in $\triangle \mathrm{COP}_{\mathrm{AP}}(P>0.05)$.

\section{Synergy analysis}

The muscle coordination pattern that elderly subjects used to stabilize the $\mathrm{COP}_{\mathrm{AP}}$ shift was examined by measuring the index of multi-M-mode synergy $(\Delta \mathrm{V})$ using the UCM analysis. The synergy index was computed as the normalized difference between the variance within the UCM and orthogonal to the UCM. Positive $\triangle \mathrm{V}$ values indicate that most variance within a given time window was within the UCM, i.e. that an average value of $\mathrm{COP}_{\mathrm{AP}}$ displacement observed within that window was stabilized by co-variation of magnitudes of the M-modes.

Figure $5 \mathrm{~A}$ shows $\Delta \mathrm{V}$ indices averaged across subjects computed for $\mathrm{COP}_{\mathrm{AP}}$ shifts during the $\mathrm{ST}_{\mathrm{CS}}$ and $\mathrm{ST}_{\mathrm{OS}}$ tasks. In general, subjects demonstrated multi-M-mode synergies stabilizing $\mathrm{COP}_{\mathrm{AP}}$ shifts $(\Delta \mathrm{V}>0)$. They all showed reproducible time changes for both ST tasks, there were relatively minor differences in $\Delta \mathrm{V}$ between the ST tasks. A two-way ANOVA with the factors Task $\left(\mathrm{ST}_{\mathrm{CS}}\right.$ and $\left.\mathrm{ST}_{\mathrm{OS}}\right)$ and Interval (T1, T2, T3, T4 and $\left.\mathrm{T} 5\right)$ was performed to analyze possible differences in the $\Delta V_{Z}$ value (Figure 5B). The ANOVA showed significant main effect of Interval $\left[\mathrm{F}_{(4,64)}=10.194, P<0.01\right]$, whereas there were no significant main effect of Task $\left[\mathrm{F}_{(1,16)}=0.228, P>0.05\right]$; no interaction was observed between Interval and Task $\left[\mathrm{F}_{(4,64)}=0.183, P>0.05\right]$. About $100 \mathrm{~ms}$ prior to the time of toe off (time zero), there was a drop in $\Delta V_{Z}$ seen across tasks. Post-hoc analyses revealed that $\Delta V_{Z}$ were significant greater for $\mathrm{T} 1, \mathrm{~T} 2, \mathrm{~T} 3, \mathrm{~T} 4$ than $\Delta \mathrm{V}_{\mathrm{Z}}$ for T5 $(P<0.05)$.

\section{Discussion}

The main finding of the experiment is that, when stepping over an obstacle, the older adults were capable of stabilizing the $\mathrm{COP}_{\mathrm{AP}}$ coordinate based on co-varied involvement of the M-modes. The synergy index $(\Delta \mathrm{V})$ changes in the elderly emerged later $\left(100 \mathrm{~ms}\right.$ prior to $\left.\mathrm{t}_{0}\right)$ and its magnitude was smaller as compared to that reported in younger persons. This result indicates that aging is associated with a preserved ability to explore the flexibility of the M-mode compositions but a decrease ability to use multi-M-mode synergies following a predictable perturbation.
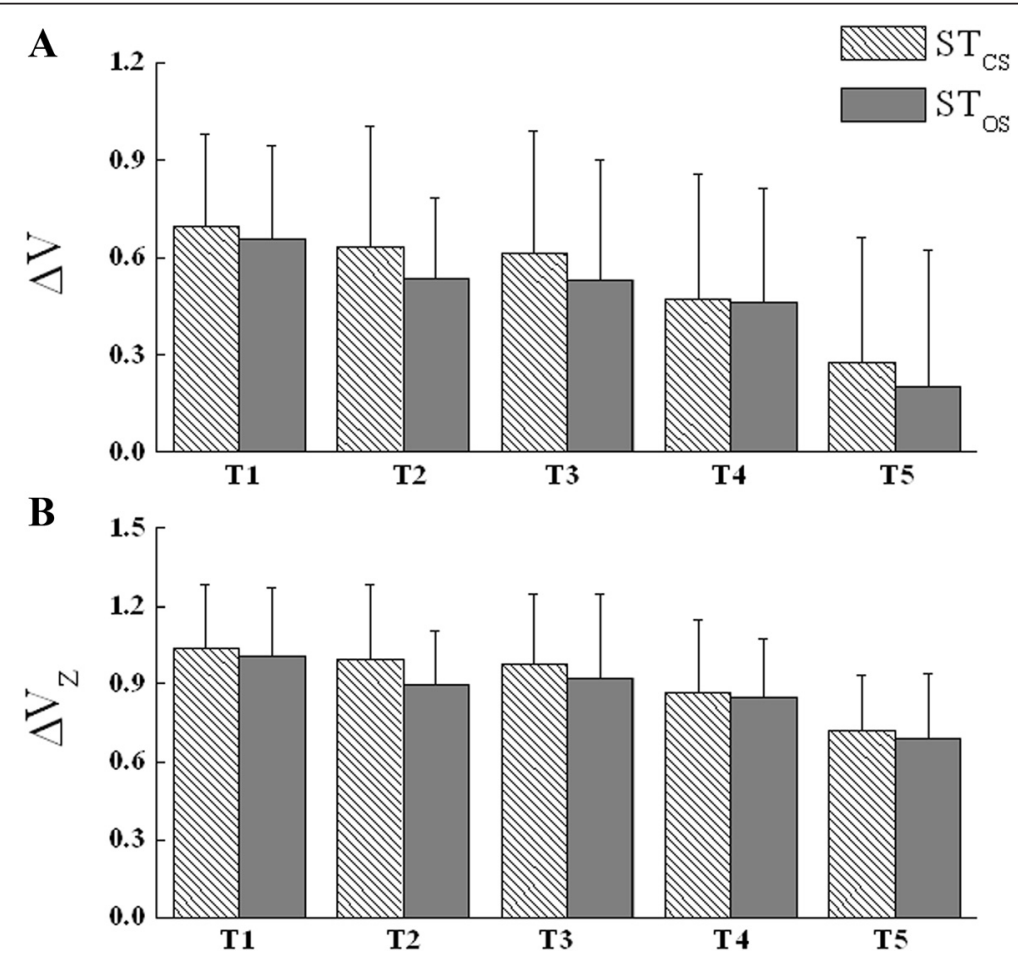

Figure 5 Mean across subjects \pm standard deviation of $\Delta V$ (panel $A)$ and $\Delta V_{Z}$ (panel B) indices for the control of the COP AP $_{A \text { ift. }}$ Adjacent pairs of bars represent the ST $T_{C S}$ (left, stripped bars) and ST OS (right, gray filled bars) tasks. $\Delta \mathrm{V}$ indices and $\Delta \mathrm{V}_{Z}$ indices were averaged over five $100 \mathrm{~ms}$ time intervals starting $500 \mathrm{~ms}$ prior to t0 and ending up at t0. 
Several recent studies suggested that postural stability involved multi-muscle synergies, especially when performing whole-body actions [7-9,20,21]. Those studies confirmed that the existence of multi-muscle synergies in a variety of everyday actions associated with the activities of daily living (ADL). The purpose of synergies has been assumed to ensure adequate mechanical conditions for maintaining optimal postural control in upright stance. Stepping over an obstacle is not an ADL but it may hamper ADL. A decline in obstacle crossing performance with advancing age may be implicated in the higher incidence of trips and stumbles in older adults [22]. Due to the changes in postural control with age [13,23-26], it is feasible that age-related changes in multi-muscle synergies can lead to trip-related falls. Yet much less attention has been paid to the ability to utilize synergic multi-muscle control of vertical posture in older persons.

When making a step with obstacle, coordinated changes in anticipatory activation of the leg and the trunk muscles are observed prior to the stepping foot takeoff. In the current study, a reciprocal M-mode was seen in the distal leg muscles of older adults in both the $\mathrm{ST}_{\mathrm{CS}}$ and $\mathrm{ST}_{\mathrm{OS}}$ tasks. Within this M-mode, dorsal muscles were generally activated whereas ventral muscles were inhibited. In the $\mathrm{ST}_{\mathrm{OS}}$ task, the proximal muscles showed more of co-contraction M-mode at the knee and hip joints. This finding suggests that the older adults chose to use a reciprocal strategy to control the ankle joint while increase the stiffness of the other joints for stabilizing the COP displacement when dealing with the instability. Indeed, similar strategies of increasing joint stiffness with co-contraction of thigh and trunk muscles under challenging postural conditions have been previously reported in healthy young and old individuals $[5,8,12,13,15,20,21,25,27,28]$ and in individuals with neurological disorders $[29,30]$ who deliberately use muscle co-contraction trading efficacy for safety. Taken together with the literature, the observed adjustments in the composition of M-modes reflect the preserved ability of elderly persons to explore the flexibility of the mechanically redundant multi-muscle system and find different solutions for compensating for the declined ability to maintain upright posture.

The coordination of multiple muscles about the longitudinal axis of the body contributes to postural stability has been shown to involve the use of muscle redundancy by the central nervous system (CNS) [5,7-10]. These studies suggest that the CNS takes advantage of muscle redundancy to stabilize the COP success by allowing for flexible combinations of redundant degrees of freedom. It should be noted that the capability to produce varied solutions to a postural task leads to movement variability. Successful postural performance requires its variability has no effect on the performance variable under consideration. The use of the uncontrolled manifold (UCM) analysis allows to separate M-mode variance into a component that reflects flexibility in stabilizing the COP ("good" variance within the UCM subspace) from a component that leads to variability of the COP ("bad" variance within the orthogonal subspace). As such, UCM analysis quantifies the structure of movement variability. The index of $\mathrm{M}$-mode co-variation $(\Delta \mathrm{V})$ reflects the relative amounts of "good" and "bad" variance in the M-modes space. If the synergy index $(\Delta \mathrm{V})$ is close to ' +1.33 ' (see equation 4 in the Methods), most M-mode variance reflects the use of muscle redundancy to stabilize the $\mathrm{COP}_{\mathrm{AP}}$ shift [31]. In the current study, the pattern of $\Delta \mathrm{V}$ change was similar to a pattern reported in a similar study of young subjects [5]. However, in the study of younger persons, the magnitude of $\Delta \mathrm{V}$ drop was significant $200 \mathrm{~ms}$ prior to the time of heel off of the stepping leg and its magnitude was about +0.6 . In the current study, $\Delta \mathrm{V}$ changes in the elderly emerged later (100 ms prior to $\left.t_{0}\right)$ and their magnitude $(+0.2)$ was about one-third of that reported in younger persons. The findings of smaller and delayed anticipatory synergy adjustments (ASAs) resemble closely the observations of preparation to a step made under the reaction time instruction in the elderly [15]. In both studies, ASAs to a self-triggered perturbation could be generated by the elderly subjects, but these adjustments were smaller and closer in time to heel off. These results suggest that although ASAs are delayed and reduced in magnitude with aging, the ability to utilize multi-M-mode synergies is largely preserved during preparation to making a step with obstacle.

Our experimental design had an inherent limitation of the number of EMG channels we could record simultaneously. We have decided to record muscles only on the stepping leg. It is possible that the supporting leg could also be important for giving valuable information which may not have been accounted for considering the changes in stepping strategy in elderly when making a step with obstacle. We will try to overcome it in future studies.

\section{Conclusions}

Postural adjustments prior to step initiation with obstacle represent a particular example of anticipatory actions; understanding adjustments on such actions to changes in the external conditions would be important for better understanding the anticipatory motor control processes with aging. Our study reveals that aging is associated with a preserved ability to explore the flexibility of the M-mode compositions but a decrease ability to use multi-M-mode synergies following a predictable perturbation. The results provide a foundation for investigating the role of training in improving the composition of M-modes and patterns of their co-variation with respect to important performance variables of postural control in older adults. 


\section{Abbreviations}

AP: Anterior-posterior; BF: Biceps femoris; COP: Center of pressure; ES: Erector spinae; GL: Lateral head of gastrocnemius; GM: Medial head of gastrocnemius; PCA: Principal component analysis; RA: Rectus abdominis; RF: Rectus femoris; SOL: Soleus; ST: Semitendinosus; TA: Tibialis anterior; UCM: Uncontrolled manifold; VL: Vastus lateralis.

\section{Competing interests}

The authors declare that they have no competing interests.

\section{Authors' contributions}

YW conceived of the study, participated in its design and setup of the study, performed data collection, preparations and statistical analyses, and drafted the manuscript. KW participated in the design, setup and coordination of the study. TA participated in the results interpretation. All authors read and approved the final manuscript.

\section{Acknowledgment}

This work was supported by the National Natural Science Foundation of China [grant \#31371207] and the Natural Science Foundation of Tianjin [grant \#14JCYBJC43300].

\section{Author details}

${ }^{1}$ Tianjin Key Lab of Exercise Physiology and Sports Medicine, Department of Health and Exercise Science, Tianjin University of Sport, 51 Weijin South Street, Hexi District, Tianjin 300381, China. ${ }^{2}$ Institute of Sports and Health Science, 3-10-31, Kagamiyama, Higashi-hiroshima, Hiroshima 739-0046, Japan. ${ }^{3}$ Department of Rehabilitation Science, Faculty of Health Sciences, Hokkaido University, N12-W5, Kita-ku, Sapporo 060-0826, Japan.

\section{Received: 25 October 2014 Accepted: 28 January 2015}

\section{Published online: 04 February 2015}

\section{References}

1. Schrodt LA, Mercer VS, Giuliani CA, Hartman M. Characteristics of stepping over an obstacle in community dwelling older adults under dual-task conditions. Gait Posture. 2004:19:279-87.

2. Hahn ME, Lee HJ, Chou LS. Increased muscular challenge in older adults during obstructed gait. Gait Posture. 2005;22(4):356-61.

3. Chou LS, Kaufman KR, Brey RH, Draganich LF. Medio-lateral motion of the center of mass during obstacle crossing distinguishes elderly individuals with imbalance. Gait Posture. 2003;18(3):125-33.

4. Huang SC, Lu TW, Chen HL, Wang TM, Chou LS. Age and height effects on the center of mass and center of pressure inclination angles during obstacle-crossing. Med Eng Phys. 2008;30(8):968-75.

5. Wang Y, Watanabe K, Asaka T, Wan FT. Muscle synergies in preparation to a step made with and without obstacle. Eur J Appl Physiol. 2014;114(12):2561-9.

6. Krishnamoorthy V, Latash ML, Scholz JP, Zatsiorsky VM. Muscle synergies during shifts of the center of pressure by standing persons. Exp Brain Res. 2003:152:281-92

7. Wang Y, Zatsiorsky VM, Latash ML. Muscle synergies involved in shifting the center of pressure while making a first step. Exp Brain Res. 2005;167(2):196-210.

8. Asaka T, Wang Y, Fukushima J, Latash ML. Learning effects on muscle modes and multi-mode postural synergies. Exp Brain Res. 2008;184(3):323-38.

9. Klous M, Mikulic P, Latash ML. Two aspects of feed-forward postural control: anticipatory postural adjustments and anticipatory synergy adjustments. J Neurophysiol. 2011;105(5):2275-88.

10. Krishnan V, Aruin AS, Latash ML. Two stages and three components of the postural preparation to action. Exp Brain Res. 2011;212(1):47-63.

11. Frank JS, Patla AE. Balance and mobility challenges in older adults: implications for preserving community mobility. Am J Prev Med. 2003;25:157-63.

12. Nagai K, Yamada M, Uemura K, Yamada Y, Ichihashi N, Tsuboyama T. Differences in muscle coactivation during postural control between healthy older and young adults. Arch Gerontol Geriatr. 2011;53:338-43.

13. Bleuse S, Cassim F, Blatt JL, Labyt E, Derambure P, Guieu JD, et al. Effect of age on anticipatory postural adjustments in unilateral arm movement. Gait Posture. 2006;24:203-10.

14. Kanekar N, Aruin AS. The effect of aging on anticipatory postural control Exp Brain Res. 2014;232(4):1127-36.
15. Wang $Y$, Asaka T, Watanabe K. Multi-muscle synergies in elderly individuals: preparation to a step made under the self-paced and reaction time instructions. Exp Brain Res. 2013;226(4):463-72.

16. Nachshon I, Denno D, Aurand S. Lateral preference of hand, eye and foot: relation to cerebral dominance. Int J Neurosci. 1983;18(1-2):1-9.

17. Wang $Y$, Watanabe K. Angular movements of the trunk and pelvis when stepping over obstacles of different heights. Res in Sports Med. 2003:11(4):219-34.

18. Wang $Y$, Watanabe $K$. The relationship between obstacle height and center of pressure velocity during obstacle crossing. Gait Posture. 2008;27(1):172-5.

19. Winter DA, Prince F, Frank JS, Powell C, Zabjek KF. Unified theory regarding A/P and M/L balance in quiet stance. J Neurophysiol. 1996;75:2334-43.

20. Wang $Y$, Asaka T. Muscle synergies involved in shifts of the center of pressure while standing on a narrow support. Brain Res Bull. 2008;76(1-2):16-25.

21. Klous M, Mikulic $P$, Latash ML. Early postural adjustments in preparation to whole-body voluntary sway. J Electromyogr Kinesiol. 2012;22(1):110-6.

22. Overstall PW, Extonsmith AN, Imms FJ, Johnson AL. Falls in elderly related to postural imbalance. BMJ. 1977;1(6056):261-4.

23. Horak FB. Postural orientation and equilibrium: what do we need to know about neural control of balance to prevent falls? Age Ageing. 2006:35 Suppl 2:ii7-ii11.

24. Sturnieks DL, St George R, Lord SR. Balance disorders in the elderly. Neurophysiol Clin. 2008:38:467-78.

25. Claudino R, dos Santos EC, Santos MJ. Compensatory but not anticipatory adjustments are altered in older adults during lateral postural perturbations. Clin Neurophysiol. 2013;124:1628-37.

26. Kanekar N, Aruin AS. Aging and balance control in response to external perturbations: role of anticipatory and compensatory postural mechanisms. Age. 2014;36:1067-77.

27. Gantchev GN, Dimiytobs DM. Anticipatory postural adjustments associated with arm movements during balancing on unstable support surface. Int J Psychophysiol. 1996;22:117-22.

28. Wang Y, Zatsiorsky VM, Latash ML. Muscle synergies involved in preparation to a step made under the self-paced and reaction time instructions. Clin Neurophysiol. 2006:117:41-56.

29. Halliday S, Winter D, Frank J, Patla A. The initiation of gait in young, elderly, and Parkinson's disease subjects. Gait Posture. 1998;8:8-14.

30. Dimitrova D, Horak FB, Nutt JG. Postural muscle responses to multidirectional translations in patients with Parkinson's disease. J Neurophysiol. 2004;91:489-501.

31. Scholz JP, Schöner G. The uncontrolled manifold concept: identifying control variables for a functional task. Exp Brain Res. 1999:126:289-306.

\section{Submit your next manuscript to BioMed Central and take full advantage of:}

- Convenient online submission

- Thorough peer review

- No space constraints or color figure charges

- Immediate publication on acceptance

- Inclusion in PubMed, CAS, Scopus and Google Scholar

- Research which is freely available for redistribution 